\title{
Reproducción asexual, sexual y vigor en semillas de caracolí Anacardium excelsum (Bertero ex Kunth) Skeels en el departamento de Sucre
}

\section{Asexual, sexual reproduction and seed vigor of Caracolí Anacaridum excelsum (Bertero ex Kunth) Skeels in the Sucre department}

\author{
Salcedo M Luis ${ }^{1}$ Biólogo, Pérez P Yesica ${ }^{1}$ Biólogo, Millán R Euriel² M.Sc.
}

1Universidad de Sucre, Departamento de Biología y Química,
Grupo de Investigación GRESA, Sincelejo, Sucre, Colombia.
2Universidad de Sucre, Departamento de Ingeniería Agrícola,
Grupo de Investigación GRESA, Sincelejo, Sucre, Colombia.

Keywords:

Anacardium excelsum;

dry forest;

Colombia;

germination;

reproduction;

growth rate;

pre-germinative treatment.

\begin{abstract}
The dry tropical forest in Colombia is in constant decline and deforestation of their coverage, Anacardium excelsum is a representative in this forest, but is almost threatened at the nationwide. Given this and its importance as a timber tree the objective of the research was to find the best form of reproduction, sexual or asexual, and determine the vigor and the best pre-germinative treatment to break dormancy of seeds. Seven different pre-germinative treatments and three with hormone solution for rooting cuttings were performed. Scarification with a scalpel and immersion in $\mathrm{HCl}$ for 5 minutes had a higher percentage of germination, most plants germinated before 4 days after being planted, and showed a growth rate between 3 and $4 \mathrm{~cm}$ per day in the first week. With respect to the asexual reproduction, in no of the stakes was obtained formation of roots and buds. Sexual reproduction proved to be the best method, and the pre-germinative scarification treatment with scalpel was the one with the highest percentage of germination.
\end{abstract}

\section{Resumen}

El bosque seco tropical en Colombia se encuentra en disminución y deforestación constante de su cobertura, Anacardium excelsum es un representante en este tipo de bosque, sin embargo se encuentra casi amenazada a nivel nacional. Teniendo en cuenta este aspecto y su importancia como especie maderable, el objetivo de la investigación fue hallar la mejor forma de reproducción, sexual o asexual, y determinar el vigor y el mejor tratamiento pre-germinativo para romper la latencia de las semillas. Se realizaron siete tratamientos pre-germinativos y tres con solución de hormona para enraizamiento de estacas. La escarificación con bisturí e inmersión en $\mathrm{HCl}$ por 5 minutos presentaron un mayor porcentaje de germinación, la mayor parte de las plantas germinaron antes de los 4 días de haber sido sembradas, y presentaron una tasa de crecimiento entre 3 y $4 \mathrm{~cm}$ por día en la primera semana. Con respecto a la reproducción asexual, en ninguna de las estacas se obtuvo formación de raíces y yemas. La reproducción sexual resultó ser el mejor método, y el tratamiento pre-germinativo de escarificación con bisturí fue el que presento mayor porcentaje de germinación. 


\section{Introducción}

Según el Libro Rojo de las especies amenazadas en Colombia (CÁRDENAS y SALINAS, 2007), Anacardium excelsum (familia Anacardiaceae) es una especie que podemos identificar en los bosques secos tropicales y que presenta grado de amenaza en un nivel NT (casi amenazada), por lo tanto, es de vital importancia buscar estrategias, como las diferentes formas de su propagación, ya sea con semillas o yemas para iniciar un plan de reforestación de dicha especie. También es conocida con las sinonimias de Rhinocarpus excelsa (Bertero ex Kunth) y Anacardium rhinocarpus D.C, reconocida con nombres comunes como: Caracolí, Aspavé, Espavé, Ispavé, Marañón de Monte y Merey. Es una especie que se encuentra distribuida geográficamente desde Costa Rica, Cuba, Panamá, Guyana, Brasil, Venezuela hasta Ecuador, y en Colombia se halla en los departamentos de Valle del Cauca, Cauca, Santander, Tolima, Huila, Chocó, el Valle Seco del Magdalena, Región de Urabá, Magdalena Medio y la Amazonía Colombiana (BERNAL et al., 2014).

En su estado adulto $A$. excelsum puede llegar a medir hasta $45 \mathrm{~m}$ con un DAP de $3 \mathrm{~m}$, libre de ramas en los primeros $15 \mathrm{~m}$; sus hojas son grandes y coriáceas, simples y alternas, agrupadas al final de las ramas, en su interior la corteza es de color rosado, y resinosa con olor similar a la trementina; sus semillas son grandes y tóxicas, de dimensiones $2.0-2.5 \mathrm{~cm}$ x $1.0-$ $1.5 \mathrm{~cm}$; su duramen es de color marrón rojizo; superficie brillante, sin olor ni sabor; y la madera es blanda y liviana (ALEGRÍA, 2012). Siendo uno de los árboles más altos de toda Sur América, presenta un Incremento Corriente Anual (ICA) en

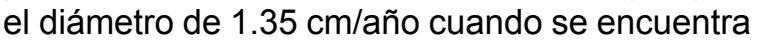
en ambientes parecidos al bosque seco tropical, la mayor parte de su crecimiento la alcanzará al llegar a los 88 años aproximadamente, pero que se hace aprovechable para uso maderable con tan solo tener entre 20-25 años. LOZANO et al. (2012) afirman que esta planta presenta un crecimiento relativamente rápido y puede ser utilizada con propósitos de reforestación protectora o comercial.

Estudios fitoquímicos han demostrado que $A$. excelsum presenta compuestos químicos como el 2-(1, 1 dimetiletil)-4-(1, 1, 3, 3-tetrametil-butil) fenol y el 2,2'-metilenbis (6-(1,1-dimetil)-4-etil- fenol, que se presentan con mayor abundancia en la corteza y en la semilla y poseen potentes propiedades antimicrobianas y antisépticas resultando en un tipo de germicida activo que puede inhibir la actividad de bacterias gram positivas como lo son Staphylococcus aureus y Bacillus subtilis. Algunos de los compuestos fenólicos presentes en la planta pueden resultar tóxicos para el hombre, pero los encontrados en su fruto son fácilmente rotos por acción de la temperatura al momento de la cocción y pueden ser utilizados como alimento (CELIS et al., 2011). Esta presencia de fenoles en las semillas es característica del género Anacardium, teniendo como ejemplo el marañón ( $A$. occidentale) que contiene aceites ricos en fenoles en la cubierta de su semilla utilizados para producir adhesivos, pinturas, plásticos y además presentan algunas propiedades terapéuticas (VIT, 2003).

A nivel departamental, la especie $A$. excelsum presenta el mayor índice de importancia en el municipio de Toluviejo - Sucre, debido principalmente a su endemismo y adaptaciones; por lo que debe ser tenida en cuenta para planes de ecoturismo y conservación, de manera que su supervivencia sea segura (SAMPEDRO et al., 2013). Algunos estudios han demostrado la importancia comercial de esta especie (BARRETO et al., 2007), en la Sierra Nevada de Santa Marta (Colombia), se enfocaron en aislar y evaluar el efecto de la inoculación de biopreparados con base en Rizobacterias promotoras del crecimiento vegetal (RPCV) exclusivos de $A$. excelsum; e investigaciones dirigidas por URREA y SEQUEDA (2012) evaluaron extractos etanólicos de $A$. excelsum para la preservación de alimentos.

Existen diversas técnicas de tratamientos pregerminativos que ayudan a romper la dormancia de las semillas, como ejemplos de estos se pueden citar la escarificación mecánica y química, los cuales han sido utilizados en diversas especies como Garcinia kola (MATIG et al., 2007); Tectona grandis (OFFIONG et al., 2010); Acacia tortilis y Acacia oerfota (ABARI et al., 2011); Afzelia africana (AMUSA, 2011); Byrsonima crassifolia, Cedrela odorata, Guazuma ulmifolia, Inga inicuil, Psidium guajava, Swietenia macrophylla y Tabebuia rosea (CHAN et al., 2012); Leucaena lanceolata (ROMÁN et al., 2013). El objetivo del presente estudio fue determinar el método 
de propagación más eficaz, asexual o sexual y vigor de semillas, para la especie Anacardium excelsum.

\section{Materiales y métodos}

El estudio se realizó en el invernadero de la Universidad del Sucre, ubicado en la ciudad de Sincelejo, ubicada a $9^{\circ} 19^{\prime} 01^{\prime \prime}$ de latitud norte y $75^{\circ} 23^{\prime} 16.39^{\prime \prime}$ de longitud oeste del meridiano de Grenwich; la cual se encuentra enmarcada en una zona ecológica de bosque seco tropical, con una altitud de $182 \mathrm{msnm}$ y una pluviosidad anual media desde $1000 \mathrm{~mm}$ para zonas menos húmedas en el norte, hasta $2800 \mathrm{~mm}$ en zonas lluviosas del sur, y condiciones de piso térmico cálido con una temperatura que oscila entre $25.5^{\circ} \mathrm{C}$ y $28.7^{\circ} \mathrm{C}$ (AGUILERA, 2005; GACETA DEPARTAMENTAL DE SUCRE, 2010); y una humedad relativa promedio de $80 \%$ según la GACETA DEPARTAMENTAL DE SUCRE (2010). Fue llevado a cabo entre los meses de Junio y Julio del 2014.

Para la reproducción sexual, las semillas se recolectaron de plantas madres de Caracolí seleccionadas, ubicadas en el corregimiento de San Jacinto (con coordenadas 9'14'52.87" $\mathrm{N}$ y $75^{\circ} 27^{\prime} 02.02$ " O) en los Montes de María Sucre, Colombia. Estas fueron lavadas con agua y alcohol y se enjuagaron cuatro veces con agua potable. La selección de las semillas se realizó de acuerdo al tamaño y peso, descartando las que presentaron rasgaduras o agujeros y aquellas que flotaron al ser sumergidas en agua. El secado se efectuó a temperatura ambiente bajo sombra, durante una hora, con el objeto de eliminar el exceso de humedad en la cubierta de la semilla, evitando su deshidratación. Luego se colocaron dentro de una bolsa plástica y se refrigeraron a temperatura de $10 \pm 1^{\circ} \mathrm{C}$ por 20 días (PEROZO et al., 2006).

Luego las semillas se sometieron a diferentes tratamientos, como lo son escarificación mecánica con lija, escarificación mecánica con bisturí, escarificación química con $\mathrm{HCl}$ por 5 y 10 minutos, además de eso se contó con un testigo. Se sembraron un total de 50 semillas para todas las pruebas, evaluándose capacidad de germinación y vigor germinativo como lo hicieron MÁPULA et al.
(2008), siendo el vigor de germinación el tiempo en el cual se alcanza la mayor tasa de germinación, se obtuvo calculando y comparando los porcentajes de semillas germinadas obtenidos en cada día de observación (BONNER y KARRFALT, 2008).

Para la reproducción asexual, en la selección y colecta de las estacas se tuvo en cuenta el estado vegetativo de los árboles, según VALLEJOS et al. (2010), un árbol plus es un espécimen arbóreo con un fenotipo sobresaliente, sinónimo de un árbol élite, que tiene características fenotípicas y genotípicas sobresalientes, que hacen que sea el perfecto para realizar estos ensayos, sin embargo fue muy difícil encontrar arboles elite como tal de esta especie y se debió contar con lo mejor posible. Se tuvo en cuenta que los arboles no se hallaran en estado de senescencia o fructificación, debido a que en estas fases en algunas especies se produce desecación y el crecimiento de los meristemos se reduce (AZCÓN y TALON, 2013). Se colectaron un total de 45 estacas provenientes de las zonas más apicales, y secciones intermedias (semiduras y blandas) por su totipotencia y tejidos más tiernos; su diámetro oscilo entre 1.0 y $2.0 \mathrm{~cm}$. y su longitud no superó los $25 \mathrm{~cm}$., siempre contando con dos o más nudos en las estacas. Estas fueron mantenidas en papel húmedo hasta el momento de llevarlas al vivero.

Para la propagación asexual por estacas, se utilizaron 5 tratamientos para diferentes tiempos. Las estacas fueron sumergidas en agua de grifo, agua destilada y solución de hormona (Ácido Indol-Butirico) en concentraciones de 100, 250 y 500 ppm por un tiempo de 30, 60 y 120 minutos. Antes de sumergirlas se retiraron hasta $2 \mathrm{~cm}$. de corteza en la parte basal de las estacas, con el propósito de que se permita una mayor absorción de las soluciones.

El sustrato que se utilizó para sembrar tanto las semillas como las estacas tuvo las mismas características. Se preparó con suelo natural, arena y lombriabono (ver Tabla 1 los componentes de lombriabono) en proporciones 2:1:1; mezclado homogéneamente. Para su desinfección se utilizó una solución de formaldehido al $0.4 \%$, cubriéndose con plástico oscuro durante tres días y luego saturado con agua de grifo par su lavado y así eliminar restos del formaldehido (GUNACTI y ERKILIC, 2013). 
Tabla 1. Características específicas del lombriabono utilizado para la preparación del sustrato.

\begin{tabular}{cc}
\hline COMPONENTE & PORCENTAJE \\
\hline Nitrógeno Total & $2 \%$ \\
Fosforo Total (P2O5) & $1 \%$ \\
Potasio Total (K2O) & $2 \%$ \\
Calcio total $(\mathrm{CaO})$ & $4 \%$ \\
Magnesio Total (MgO) & $1 \%$ \\
Carbono oxidable orgánico total & $23.69 \%$ \\
Humedad & $24 \%$ \\
Capacidad de intercambio catiónico & $72.52 \mathrm{meg} / 100 \mathrm{~g}$ \\
Capacidad de retención de humedad & $164.0 \%$ \\
pH & $6.57 \%$ \\
Densidad & $0.6 \mathrm{~g} / \mathrm{c}$ \\
Conductividad eléctrica & $15.84 \mathrm{mS} / \mathrm{cm}$ \\
Salmonella & Ausente en $25 / \mathrm{ml}$ \\
Enterobacterias Totales & Ausente \\
\hline
\end{tabular}

Para determinar el desarrollo de cada individuo periódicamente se evaluaron características como: altura, número de nudos, número de hojas. Al final de la experimentación se calculó área foliar y área de raíz con ayuda de papel milimetrado y planímetro digital.

Para los análisis de datos, se realizaron graficas del porcentaje de germinación en cada tratamiento y tasa de crecimiento promedio de las plantas germinadas en el programa Microsoft Office Excel versión 2010. Se hicieron análisis estadísticos de ANOVA y comparación de medias con prueba Tukey mediante el software $R$ versión 3.1.1, para un nivel de significancia de $p<0.05$; y tres repeticiones por tratamiento.

\section{Resultados}

Al final de los 35 días de observación en el invernadero, se obtuvieron los resultados presentados en la Tabla 2, teniendo el número de plantas germinadas, el número de nudos y hojas formadas así como el área de la raíz y la parte aérea de cada planta. La gran parte de las semillas empezaron a germinar entre los dos y cuatro días de estar dentro del sustrato en el invernadero.

Teniendo en cuenta los resultados presentados en la tabla 2 y la figura1, se puede notar claramente que se obtuvo un mayor porcentaje de semillas germinadas mediante el tratamiento de escarificación con bisturí (60\%), seguido del tratamiento de sumergir en solución de $\mathrm{HCl}$ por 5 minutos (40\%). En los tratamientos donde hubo un menor número de semillas germinadas fueron el testigo y la escarificación con lija, mostrando no tener un efecto apreciable sobre el rompimiento de la dormancia en las semillas. El agua destilada en conjunto con el agua caliente y la solución de $\mathrm{HCl}$ por 10 minutos, presentaron similares porcentajes en cuanto a la germinación (20\%), mostrando efectos semejantes sobre la testas de las semillas.

Tabla 2. Resultados del número de plantas germinadas en cada tratamiento y sus respectivos datos de crecimiento al finalizar los 35 días.

\begin{tabular}{cccccc}
\hline TRATAMIENTO & $\mathbf{N}^{\circ}$ & NN & NH & AR & AP \\
\hline Testigo & 1 & 12 & 10 & 256 & 1440 \\
& 1 & 11 & 9 & 138 & 1191.9 \\
& 2 & 11 & 9 & 160 & 1058.5 \\
Esc con bisturí & 4 & 11 & 9 & 97.75 & 731 \\
& 5 & 10 & 8 & 45 & 445.3 \\
Esc con lija & 1 & 13 & 11 & 175 & 465.5 \\
Agua destilada & 1 & 13 & 11 & 154 & 1283.75 \\
& 2 & 13 & 11 & 78 & 1067.8 \\
Agua caliente & 1 & 11 & 9 & 99 & 1111.5 \\
& 2 & 11 & 9 & 55 & 563.75 \\
& 1 & 12 & 10 & 192 & 1122 \\
HCl por 5 min. & 3 & 11 & 9 & 182.25 & 940.5 \\
& 4 & 10 & 8 & 112.5 & 460 \\
HCl por 10 min. & 1 & 11 & 9 & 105 & 945.5 \\
& 2 & 6 & 4 & 4.5 & 834.75 \\
\hline
\end{tabular}

Esc= Escarificación $; \mathrm{N}^{\circ}=$ Planta $\mathrm{N}^{\circ} ; \mathrm{NN}=\mathrm{N}^{\circ} \mathrm{DE}$ NUDOS; $\mathrm{NH}=\mathrm{N}^{\circ}$ DE HOJAS; $\mathrm{AR}=$ AREA DE LA RAIZ $\left(\mathrm{cm}^{2}\right)$; AP = AREA DE LA PARTE AEREA $\left(\mathrm{cm}^{2}\right)$.

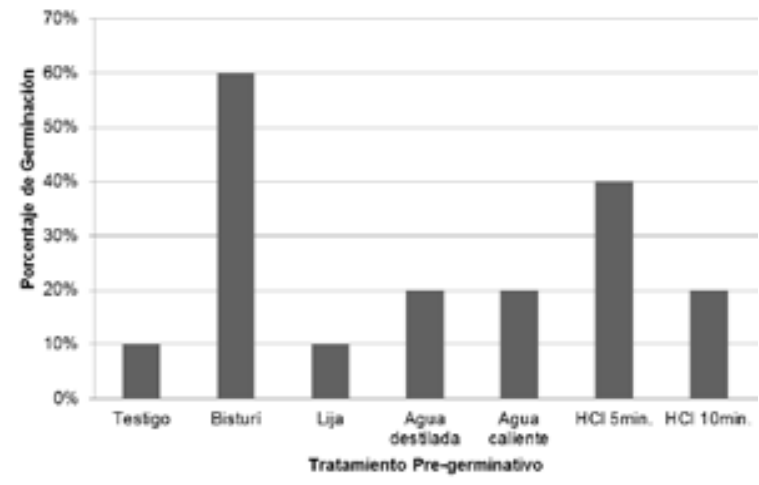

Figura 1. Porcentaje de semillas germinadas en cada uno de los diferentes tratamientos. 
De las mediciones tomadas cada tres o cuatro días de experimentación, se pudo obtener una tasa de crecimiento promedio de las plantas formadas en los diferentes tratamientos, para esta grafica se consideraron las plantas que lograron sobrevivir hasta los 35 días, por ello la plántula $\mathrm{N}^{\circ} 2$ obtenida en el tratamiento de $\mathrm{HCl}$ por 10 minutos no se tuvo en cuenta debido a que luego de germinar presentó un atrofio en su desarrollo lo que condujo a un creciendo anormal y posterior muerte. Mostrándose en la figura 2 que al pasar los primeros ocho días después de la siembra, todas las plantas que se formaron habían germinado, las primeras germinaron a los dos días y las últimas antes de los ocho días. La máxima tasa de germinación diaria fue $11 \%$, (vigor germinativo) y se presentó entre los días 2 y 4 . Las plantas pertenecientes a los tratamientos testigo y escarificación con lija obtuvieron un mayor crecimiento total al transcurrir los 35 días comparado con el resto, 30 y 29.8 $\mathrm{cm}$ respectivamente; seguidas de las plantas de escarificación con bisturí y la solución de $\mathrm{HCl} 5$ minutos. Las mayores tasas de crecimiento se presentaron en los primeros ocho días luego de germinar, encontrándose entre 3 y $4 \mathrm{~cm}$ por día. Al pasar los días la tasa de crecimiento disminuyó, manteniéndose estable en todas las plantas.

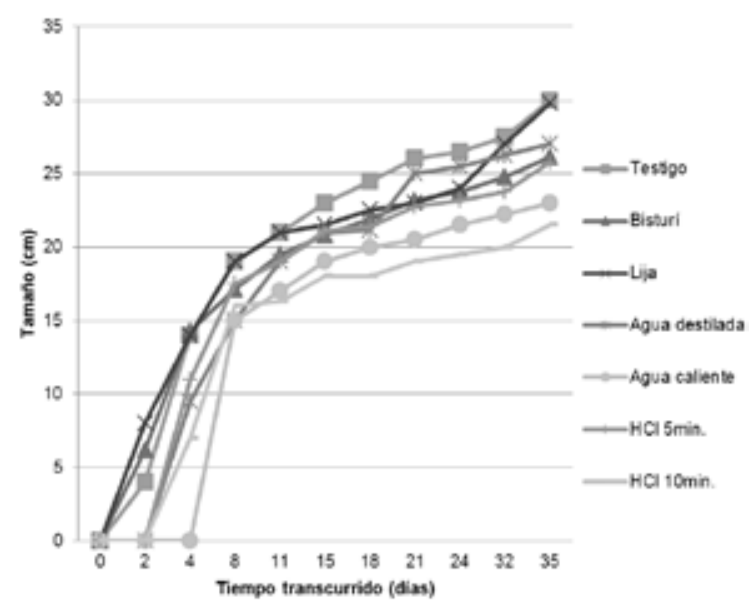

Figura 2. Tasa de crecimiento promedio de las plantas que lograron germinar y desarrollarse al pasar los 35 días de observación.

Los resultados del número de hojas, áreas de la raíz y área de la parte foliar de las plantas producidas en los diferentes tratamientos, son presentados en la Tabla 3. Hubo diferencias estadísticamente significativas por la prueba de Tukey, las plantas pertenecientes a los tratamientos de escarificación con lija y agua destilada obtuvieron mayor número de hojas (11), seguidos del testigo (10) y el resto de tratamientos con 9 o menos hojas. En cuanto al área de la raíz, la planta obtenida en el tratamiento testigo tuvo un mayor desarrollo que las demás, seguido de los tratamientos de escarificación con lija y la solución de $\mathrm{HCl}$ por 5 minutos.

Tabla 3. Medias del número de hojas, área de las raíces y área de la parte aérea de las plantas.

\begin{tabular}{|c|c|c|c|}
\hline \multirow{2}{*}{ TRATAMIENTO } & \multicolumn{3}{|c|}{ MEDIAS* } \\
\hline & NH & AR & AP \\
\hline Testigo & $10 \mathrm{~b}$ & $256 a$ & $1440^{a}$ \\
\hline Escarificación con Bisturí & $8.66 c$ & $129.5 c$ & $795.3 c$ \\
\hline Escarificación con lija & $11 a$ & $144 b$ & $942.9 \mathrm{c}$ \\
\hline Agua destilada & $11 a$ & $116 c$ & $1175.8 \mathrm{~b}$ \\
\hline Agua caliente & $9 c$ & $77 c$ & $837.6 \mathrm{c}$ \\
\hline $\mathrm{HCl}$ por $5 \mathrm{~min}$. & $8.75 c$ & $147.9 \mathrm{~b}$ & $867 c$ \\
\hline $\mathrm{HCl}$ por $10 \mathrm{~min}$. & $9 c$ & $110 c$ & $834.75 c$ \\
\hline P - Value Obtenido & 0.01059 & 0.0406 & 0.0034 \\
\hline
\end{tabular}

*Letras diferentes en la misma línea vertical, para la misma característica, indican diferencias estadísticamente significativas con $\mathrm{p}<0.05$ por la prueba Tukey. $\mathrm{NH}=\mathrm{N}^{\circ}$ DE HOJAS ; AR=AREA DE LARAIZ $\left(\mathrm{cm}^{2}\right)$; AP = AREADE LAPARTE AEREA $\left(\mathrm{cm}^{2}\right)$

Lo concerniente al área de la parte foliar en las plantas, nuevamente la planta del tratamiento testigo presentó un mayor desarrollo aéreo con diferencias significativas referente a los demás tratamientos, seguido del tratamiento con agua destilada.

En lo que corresponde a la reproducción asexual, al pasar los 35 días de experimentación las estacas de $A$. excelsum presentaron desecación y no formación de yemas ni raíces en todas las concentraciones de la fitohormona Acido IndolButirico y los tiempos de sumersión en los que se realizó la investigación.

\section{Discusión}

El tiempo de inicio de germinación fue muy similar en todas las semillas, la mayoría germinó a los dos días de siembra, aunque WILLENBORG et al. (2005) y PÉREZ (2013) proponen que siempre existirá una diferencia en el tiempo de germinación entre una semilla y otra debido a su fisiología y contenido genético totalmente 
individual, en este caso la diferencia en el tiempo fueron de tan solo horas o minutos, a causa de que las semillas, por provenir de árboles ubicados en la misma localidad (muy cerca el uno del otro), presenten características semejantes ya que están expuestas a ambientes similares. Teniendo en cuenta que el vigor germinativo se considera como el tiempo requerido para alcanzar la máxima tasa de germinación diaria a partir del inicio de la evaluación (BONNER y KARRFALT, 2008), esta especie presentó un bajo vigor (2 y 4 días) relativo a otras especies como lo es Pseudotsuga menziesii que presentó un tiempo de 8 y 13 días en resultados expresados por MÁPULA et al. (2008). A. excelsum tardó un tiempo mucho menor en alcanzar la mayor tasa de germinación con relación a otras plantas que se les ha evaluado el vigor germinativo, como lo son Swietenia macrophylla (ACOSTA et al., 2011); Ormosia macrocalyx, Manilkara zapota, Eugenia sp (PÉREZ et al., 2011); Pinus engelmannii (BUSTAMANTE et al., 2012); Stenocereus thurberi (PÉREZ, 2013); Myroxylon balsamum (PAYARES et al., 2014); lo que demuestra ser una especie que necesita poco tiempo para su propagación.

Con respecto al porcentaje de germinación, el mayor fue de $60 \%$ (escarificación con bisturí), resultado mucho menor con relación a trabajos realizados con otras especies vegetales como Manilkara zapota (GARCÍA et al., 2010); Hymenaea courbaril (OROZCO et al., 2010); Swietenia macrophylla (ACOSTA et al., 2011) y Leucaena lanceolata (ROMÁN et al., 2013) donde se obtuvieron porcentajes de germinación entre el 75 y el $100 \%$ de las semillas. Cabe resaltar que de todos los tratamientos pre-germinativos, la escarificación mecánica con bisturí fue el que tuvo un mejor efecto sobre el proceso de germinación debido a la eliminación de inhibidores que se encuentran concentrados en la testa de semillas (compuestos fenólicos) (SILVA, 2013).
Lo que respecta a las estacas, los resultados fueron negativos, con una formación nula de raíces y yemas sin importar la ausencia o presencia de la hormona ácido Indol-3Butirico, y tampoco la concentración a la que se encontrara debido a la presencia de compuestos fenólicos en la corteza de $A$. excelsum como el 2-(1,1-dimetiletil)-4-(1,1,3,3-tetrametilbutil) fenol y el 2,2'-metilenebis(6-(1,1-dimetiletil)-4-etil-fenol (CELIS et al., 2011), y su capacidad de inactivar y/o degradar este tipo de hormonas vegetales (ACOSTA, 1981; SEQUEDA, 2008); por lo que será necesario hacer pruebas con otro tipo de hormonas para posibles resultados con estacas.

\section{Conclusiones}

Para multiplicar la especie $A$. excelsum, la reproducción sexual es la más eficaz para obtener plantas sanas y que al aplicar un tratamiento pregerminativo, la escarificación mecánica con bisturí, presenta un mayor porcentaje de germinación y en general un desarrollo normal en las plántulas. Las plantas de esta especie formadas a partir de las semillas poseen mayor tasa de crecimiento en los primeros ocho días, luego se reduce y se va haciendo constante al transcurrir de los días. La reproducción asexual no presento rizogenia (producción de raíces) o yemas caulinares y teniendo en cuenta que en las estacas testigo tampoco se formaron, no se recomienda como método para la propagación de $A$. excelsum. Se pueden realizar experimentos futuros con otras concentraciones de la hormona Ácido Indolbutirico o hacerlo con soluciones de hormonas diferentes que no puedan ser inactivadas por los compuestos químicos que contiene esta planta en la corteza.

\section{Agradecimientos}

A la Universidad de Sucre y al doctor Javier Beltrán por permitirnos y facilitarnos el espacio en el invernadero de la Universidad para llevar a cabo la investigación. 


\section{Referencias}

ABARI, A.; HOSSEINI, M.; HOJJATI, M.; BAYAT, D. 2011. Salt effects on seed germination and seedling emergence of two Acacia species. African Journal of Plant Science 5 (1): 52-56.

ACOSTA, M. 1981. Efecto de fenoles y $\mathrm{Mn}^{2+}$ sobre la degradación de ácido índole-3-acético catalizada por perixodasa. Anales de la Universidad de Murcia 36 (1-4): 109-180.

ACOSTA, F.; ORANTES, C.; GARRIDO, E. 2011. Germinación y crecimiento de plántulas de caoba (Swietenia macrophylla King, Meliaceae) en condiciones de vivero. Lacadonia 5 (1): 13-20.

AGUILERA, M. 2005. La Economía del Departamento de Sucre: Ganadería y Sector Público. Cartagena: Banco de la República, Centro de Estudios Económicos Regionales CEER.

ALEGRÍA, J. L. 2012. Modelo Cuantitativo de la Arquitectura Arbórea, en Anacardium excelsum, en Quimbaya, Quindío, Colombia (Trabajo de pregrado, Biología). Cali: Universidad del valle. 51 p.

AMUSA, T. O. 2011. Effects of tree pre-treatment techniques on dormancy and germination of sedes of Afzelia Africana (Sm. Ex pers). Journal of Horticulture and Forestly 3 (4): 96-103.

AZCÓN, J. \& TALON, M. 2013. Fundamentos de fisiología vegetal segunda edición. Madrid: Editorial McGrawHill - Interamericana de España, S.L.

BARRETO, D.; VALERO, N.; MUÑOZ, A.; PERALTA, A. 2007. Efectos de Microorganismos Rizosféricos sobre Germinación y crecimiento Temprano de Anacardium excelsum. Zonas Aridas 11 (1): 240-250.

BERNAL, R.; GALEANO, G.; RODRÍGUEZ, A.; SARMIENTO, H.; GUTIÉRREZ, M. 2014. Nombres comunes de las plantas de Colombia [citado 10 ene 2015]. Disponible en: URL: http//www.biovirtual.unal.co/ nombrescomunes/

BONNER, F.; KARRFALT, R. 2008. The Woody Plant Seed Manual. Nueva Orleans: USDA, Forest Service.

BUSTAMANTE, V.; PRIETO, J.; MERLÍN, E.; ÁLVAREZ, R.; CARRILLO, A.; HERNÁNDEZ, J. 2012. Potencial y eficiencia de producción de semilla de Pinus engelmannii Carr., en tres rodales semilleros del estado de Durango, México. Madera y bosques 18 (3): 7-21.

CÁRDENAS, L.; SALINAS, N. 2007. Libro Rojo de plantas de Colombia. Volumen 4, Especies maderables amenazadas: primera parte. Bogotá: Instituto Amazónico de Investigaciones Científicias SINCHI - Ministerio de Ambiente, Vivienda y Desarrollo Territorial. Colombia.

CELIS, C.; GARCÍA, A.; SEQUEDA, G.; MÉNDEZ, G.; TORRENEGRA, R. 2011. Antimicrobial activity of extracts obtained from Anacardium excelsum againts some pathogenic microorganisms. Emirates Journal of Food \& Agriculture (EJFA) 23 (3): 249-257.

CHAN, J. G.; OCHOA, S.; PÉREZ, I. 2012. Germinación y sobrevivencia de especies arbóreas que crecen en suelos contaminados con hidrocarburos. Teoría y Praxis: 102-119.

GACETA DEPARTAMENTAL DE SUCRE. 2010. Plan vial departamental de Sucre 2010-2019. Sincelejo: Gobernación de Sucre. Colombia.

GARCÍA, M.; ORANTES, C.; MICELI, C.; GARRIDO, E.; PÉREZ, R. 2010. Germinación de semillas de chicozapote (Manilkara zapota (L.) P. Royen (Sapotáceas). Lacandonia 4 (1): 7-22. 
GUNACTI, H.; ERKILIC, A. 2013. Developing control strategies of potato wart disease (Synchytrium endobioticum) in Turkey. ESci Journal of Plant Pathology, 2 (2): 76-83.

LOZANO, L. A.; FRANCO, N.; BONILLA, J. L. 2012. Estimación del crecimiento diamétrico, de Anacardium excelsum (KUNTH) SKEELS, por medio de modelos no lineales, en bosques naturales del departamento del Tolima. Boletín Científico, Centro de Museos, Museo de Historia Natural 16 (1): 19-32.

MÁPULA, M.; LÓPEZ, J.; VARGAS, J.; HERNÁNDEZ, A. 2008. Germinación y vigor de semillas en Pseudotsuga menziesii de México. Ra Ximhai 4 (1): 119-134.

MATIG, O.; AOUDJI, A. K.; LINSOUSSI, C. 2007. Garcinia kola heckel seed dormancy-breaking. Applied ecology and environmental research 5 (1): 63-71.

OFFIONG, M. O.; UDOFIA, S. I.; OLAJIDE, O.; UFOT, I. N. 2010. Comparative study of pre-germination treatments and their effects on the growth of Tectona grandis (Linn. F) seedlings. African Research Review 4 (3): 368-378.

OROZCO, A.; FRANCO, N.; TABORDA, L. 2010. Evaluación de tres métodos de escarificación en semillas de Algarrobo (Hymenaea couybaril L.). Revista Investigaciones Universidad de Quindío 20: 36-41.

PAYARES, I.; MARIO, O.; MEDRANO, M.; MILLÁN, E. 2014. Germinación y desarrollo de plántulas de Myroxylon balsamum (I.) Harms en el departamento de Sucre. Colombia Forestal 17 (2): 193-201.

PÉREZ, S. B. 2013. Viviparidad, Germinación y Supervivencia en Stenocereus thurberi (Cactaceae). (Trabajo de Maestría en Ciencias). México: Colegio de Posgraduados, Institución de Enseñanza e investigación en Ciencias Agrícolas. 92 p.

PÉREZ, I.; OCHOA, S.; VERGAS, G.; MENDOZA, M.; GONZÁLEZ, N. 2011. Germinación y supervivencia de seis especies nativas de un bosque tropical de Tabasco, México. Madera y Bosques 17 (1): 71-91.

PEROZO, A.; RAMÍREZ, M.; GÓMEZ, A.; BUITRAGO, N. 2006. Germinación y caracterización morfológica de plántulas de merey (Anacardium occidentale L.) tipo Amarillo. Revista de la Facultad de Agronomía 23 (1): 17-27.

R DEVELOPMENT CORE TEAM. 2014. R: A language and environment for statistical computing. Vienna: $R$ Foundation for Statistical Computing. ISBN: 3-900051-07-0 [Programa de Ordenador] Disponible en: URL: http://www.R-project.org.

ROMÁN, M. L.; MARTíNEZ, L. A.; MORA, A.; TORRES, P.; GALLEGOS, A.; AVENDAÑO, A. 2013. Leucaena lanceolata S. Watson ssp. lanceolata, espécie forestal com potencial para ser introducida em sistemas silvopastoriles. Revista Chapingo serie Ciencias Forestales y del Ambiente: 103-114.

SAMPEDRO, A.; ÁLVAREZ, A.; DOMINGUEZ, L. M.; HERRERA, I. 2013. Especies promisorias para el ecoturismo en "Campo Aventura Roca Madre", Toluviejo-Sucre, Colombia. Revista MVZ Córdoba 18 (1): 3387-3398.

SEQUEDA, L. 2008. Actividad antioxidante de extractos totales y fracciones de la especie vegetal Anacardium excelsum (Bert. \& Balb. ex Kunth) skeel por métodos espectrofotométricos y composición de la fracción activa por CG-EM (Trabajo de Maestría). Colombia: Pontificia Universidad Javeriana. 145 p.

SILVA, M. 2013. Viabilidade de sementes de Handroanthus heptaphyllus (Vell.) Mattos armazenadas nos frutos. (Trabajo de Maestría). Brasil: Universidad Estatal Paulista "Júlio de Mesquita Filho". 70 p. 
URREA, V.; SEQUEDA, L. 2012. Evaluación de los extractos de Anacardium excelsum (Anacardiaceae) como alternativa hacia la preservación de alimentos. Vitae 9 (Suplemento 1): 394-396.

VALLEJOS, J.; BADILLA, Y.; PICADO, F.; MURILLO, O. 2010. Metodología para la selección e incorporación de árboles plus en programas de mejoramiento genético forestal. Agronomía Costarricense 34 (1): 105-111.

VIT, P. (2003). Anacardium occidentale L. Ficha botánica de interés apícola en Venezuela, No. 6 Merey. Revista de la Facultad de Farmacia 45 (1): 77-79.

WILLENBORG, C.; WILDEMAN, J.; MILLER, A.; ROSSNAGEL, B.; SHIRTLIFFE, S. 2005. Oat Germination Characteristics Differ among Genotypes, Seed Sizes, and Osmotic Potentials. Crop Science Society of America 45 (5): 2023-2029. 